\title{
Managing the elderly with dementia and frequent falls
}

\author{
Si Ching LIM* \\ Senior Consultant, Department of Geriatric Medicine, Changi General Hospital, Singapore
}

\begin{abstract}
The elderly living with dementia are increasing all over the world. Falls and fall related injuries are common among the elderly with dementia. The elderly with dementia are often more likely to sustain injuries after a fall and are more likely to have recurrent fallers. Once an elderly with dementia is admitted to an acute hospital, they are at risk of falls in the hospital because of delirium, unfamiliar environment, changes in their daily routine and caregivers. The usual fall prevention strategies recommended are ineffective among the elderly with dementia.
\end{abstract}

\section{Introduction}

The incidence of dementia worldwide continues to increase as the world's population ages. According to the Alzheimer Association International, someone in the world develops dementia every 3 seconds. There are currently close to 50 million people living with dementia and this number will reach 131.5 million by 2050 with much of the people with dementia living in the developing countries. [1] Dementia is a syndrome caused by chronic neurodegenerative diseases resulting in progressive decline in function, cognition and the emergence of neuropsychiatric symptoms.

Falls are common among the elderly, and the fallers often end up with recurrent falls. For the community dwelling elderly with dementia or cognitive impairment, their fall risk is higher compared to their peers with normal cognition, and their risks of sustaining serious injuries after falls are trebled or quadrupled. [2-3] Falls, whether injurious or otherwise, are associated with functional decline. [4] In the hospital setting, the elderly with delirium or dementia are also at risk of falls during their hospital stay. [5]

The risk factors causing falls are multiple and the more risk factors an individual has, the higher the risk. This paper aims to summarise the factors which cause falls among the elderly with dementia and interventions which are effective in reducing further falls among the elderly fallers with dementia.

\section{Factors contributing to falls among the elderly with dementia}

The human walking requires an intact motor, sensory, balance, vision and postural control to maintain an upright posture and to move safely through space. In addition, higher cognitive functions like attention, executive function, reaction time, navigation and visuospatial skills are important to navigate the environment and avoid the hazards on the path. People with dementia can have abnormalities of any of the functions mentioned. In addition, the people with dementia are often impulsive and take unnecessary risks due to their lack of insight and poor perception of the environmental hazards. [6] The factors which may predispose to falls among the elderly with dementia are summarized in (Table 1).
Table 1. Factors predisposing to falls among the elderly with dementia.

\begin{tabular}{|l|l|}
\hline Dementia & Type and severity of dementia \\
\hline Depression & Slow gait, cognitive symptoms, unstable gait \\
\hline Cognitive deficits & Attention, executive dysfunction, orientation, judgement \\
\hline Gait abnormalities & Gait speed, stride length, extrapyramidal signs \\
\hline Drugs & $\begin{array}{l}\text { Polypharmacy, anticholinergic properties, drugs which } \\
\text { cause gait instability, sedation, cognitive dysfunction, } \\
\text { orthostatic hypotension. }\end{array}$ \\
\hline Delirium & New onset of falls \\
\hline Neuropsychiatric symptoms & Wandering, restlessness, psychotropic medications \\
\hline Functional impairments & Physical restraints, assistance with ADLs. \\
\hline Urinary incontinence & Multifactorial, autonomic dysfunction, drugs. \\
\hline Visual impairment & Poor acuity, depth perception \\
\hline Medical problems & Postural hypotension, frailty, neuropathy, syncope, etc \\
\hline Environmental & Footwear, wet floor, clutter, etc \\
\hline
\end{tabular}

\section{Gait abnormalities and dementia}

\section{- Gait Apraxia}

Dementia consists of a host of diseases which lead to pathologies affecting the cerebral cortex, basal ganglia, thalamus and subcortical white matter. Most patients with dementia showed highest level gait disorders, also known as gait apraxia. The abnormalities affect the cortical-basal ganglia-Thalamocortical loop which interferes with adjustments of posture and movements in relation to the patients' needs and the environment. Gait apraxia is described by Meyer and Barron [1960] as the inability to use the lower limbs in the act of walking, in the absence of demonstrable sensory or motor deficits.

The clinical features of gait apraxia commonly observed include poorly coordinated limb movements, particularly during shifts in movements like sit to stand, initiate gait from stationery stance or turn

Correspondence to: Si Ching LIM, MB. ChB, MRCP, Senior Consultant, Department of Geriatric Medicine, Changi General Hospital, Singapore, Email: si_ching_lim@cgh.co m.sg

Key words: falls, dementia, gait abnormalities, BPSD, restraint

Received: November 04, 2017; Accepted: November 28, 2017; Published: December 02, 2017 
corners. The foot placement may be bizarre or inappropriate [crossing legs when standing or walking, foot placed too far forward]. The overall posture may look bizarre with patient leaning backwards when standing or turning to one side when sitting down. There is often hesitancy on initiation of movement and freezing after stopping. The overall performance is also highly variable depending on the environment and the emotional state of the patient, where the disturbance may be minimal in a calm and uncluttered environment and may be grossly abnormal and bizarre in another. There may be inappropriate or lack of rescue reactions when there is a sudden change in posture resulting in patient falling like a log when they are off balance, for instance. A careful observation of the patient's gait on standing up, turning, initiating, turning around and stopping will show the inconsistent features and the abnormalities are especially pronounced during changes in posture. [7]

\section{- Gait speed}

Gait speed is easily assessed in the clinical setting and has been shown to have predictive values for longer term outcome among the elderly. Timed up and go is a well recognised screening tool for measuring gait speed. Gait speed has been shown to be related to falls, frailty, hospitalization, mortality and has even been associated with dementia and mild cognitive impairment. [8-9]

Slower gait speed has been thought to predate onset of dementia by about 7 years, particularly the non-Alzheimer Disease dementia. [10] Slower gait speed is associated with subcortical white matter changes. In addition, most studies also reported cognitive deficits, urinary dysfunction, other gait abnormalities such as rigidity, gait apraxia, poor balance, high fall risk, depression, disability and higher mortality as features of subcortical white matter changes. In the LADIS Study, severity of age related white matter changes correlated with slower walking speed and poor balance. [11]

\section{- Dementia type and gait abnormalities}

Gait and balance disorders have been found to be commoner among the non-Alzheimer Dementia, such as Parkinson Disease Dementia [PDD], Vascular dementia [VaD] and Diffuse Lewy Body Dementia [DLB]. The presence of gait abnormality early in a person with dementia suggests a diagnosis of $\mathrm{VaD}, \mathrm{PDD}$ or $\mathrm{DLB}$, rather than $\mathrm{AD}$. The gait abnormalities in PDD are related to pathology in the Substantia Nigra; subcortical white matter disease in $\mathrm{VaD}$. The balance and gait abnormalities were most severe with greater levels of disability among the elderly with PDD [12-13].

Executive function and gait: The inability to walk while performing another task, such as walking while talking, has been associated with risk of falls among the elderly. The main deficit is the inability to allocate full attention between two demanding tasks, while performing one of either task. The gait changes while dual tasking and falls were more common among the institutionalized and frail elderly on multiple medications. [14]

The other consideration for inability to dual task while walking was the prioritization and complexity of the tasks. If the additional task was given a higher priority by the elderly, or in the case of a complex problem solving task while walking, this gives rise to the need to stop talking or walking while focusing on the other task. Trials used various methods for either of those task, some were simple motor tasks while others used complicated cognitive tasks requiring difficult verbal responses. The limited cognitive reserves may not be able to allocate attention appropriately to both the tasks simultaneously. The second task, if too simple might also under predict the fall risk because of a ceiling effect of the task used. Kressig RW [15] observed an increase in stride time variability when subjects were counting backwards, which was associated with falls. Comparing the genders, women are more likely to show variability in their gait while walking, especially so if they are dual tasking. The greater variability in gait during walking is associated with a higher fall risk. [16]

Assessment of the walking patterns, too varied between the trials. Walking straight ahead may be less demanding than walking pattern which requires subject to walk straight, turn around and return. Despite the heterogeneous dual task conditions used in the various studies, there is a statistically significant increase in fall risk when subjects were assessed on walking and gait while dual tasking. The more challenging walking pattern has a higher risk for falls. [14]

Depression and falls: Depression is associated with a higher fall risk among the institutionalized and community dwelling elderly. There are multiple factors where depression contributes to falls. Depression has been shown to be associated with poor physical performance and disability. Declining physical performance measured using gait speed, is proportional to the severity of depressive symptoms. Demakakos $\mathrm{P}$ showed that the association between depression and gait speed was bidirectional, such that slowed gait speed is associated with concurrent and future depressive symptoms. [17]

Depression is now recognized as a risk factor as well as a prodromal symptom of dementia. Both depression and dementia have been found to share similar neurobiological changes in the brain. Among the persons with dementia, depression is also a common co-occurrence and is often difficult to treat. [18] In addition to depression increasing fall risk, the use of antidepressants is also associated with higher fall risk, especially outdoor falls. [19]

Depression is closely associated with frailty among the elderly. Depression and frailty share overlapping symptoms like weight loss, exhaustion, physical disability and low level of physical activity. In the clinical setting, both the syndromes of frailty and depression maybe interlinked. Frailty is well known to be associated with falls, hospitalisations, disability and death. It may also be that one syndrome caused the other, for example a frail elderly developed functional decline after repeated hospitalisations, which leads to further dependency on activities of daily living, giving rise to depression; or an elderly with depression with loss of interest who develop disability after a long period of low physical activities, and subsequently develop features of frailty. [20]

Drugs and fall risk: Polypharmacy is common among the elderly, due to their multiple comorbidities. Polypharmacy increases the risk of side effects and drug-drug interactions. A meta-analysis showed that the elderly are more prone to develop adverse drug reactions requiring hospitalisations and nearly $90 \%$ of the adverse reactions are preventable. [21]

Culprit drugs which increase fall risk include psychotropics which include antipsychotics, antidepressants, sedatives/ hypnotics. According to Beer's Criteria, antipsychotics increase risk of cerebrovascular events, cognitive decline and mortality. It also causes sedation and gait abnormalities. Antidepressants are sedating, some have anticholinergic side effects causing orthostatic hypotension which increase fall risk. Benzodiazepines have longer half-life among the elderly, causing prolonged sedation, cognitive impairment and risk of delirium. Non-benzodiazepine receptor agonists like zopiclone and zolpidem have similar side effects profile to benzodiazepines, and because of their limited effectiveness and high fall risk, they are to be avoided among the elderly. 
Drugs which cause orthostatic hypotension also predispose to falls among the elderly. Commonly used drugs causing orthostatic hypotension include, alpha-1 blockers, vasodilators, cardiovascular drugs and antihypertensive drugs. Centrally acting drugs like anticonvulsants, tricyclic antidepressants, SSRIs and opioids are also recognized to increase falls and fracture risks. [22]

Delirium and falls: In the acute hospital setting, delirium was present in $96 \%$ of the elderly fallers. However, delirium is often missed and in the study by Lakatos et. al, delirium was only documented in the discharge summary in 19\% of the fallers. [23] A diagnosis of inpatient delirium which persisted after hospitalization is associated with a sixfold increase risk of falls. [24]

Any new onset of falls among the elderly should be considered as delirium and an active search for underlying causes be made, including recent changes in medications. [25]

Behavioural symptoms of dementia and falls: In addition to cognitive symptoms, behavioural and psychological symptoms [BPSD] are common among people with dementia. Behavioural symptoms which are associated with higher level of physical activities like wandering, restlessness, trying to escape, verbal disruption and attention seeking behaviors were associated with higher risk of falls. Psychological symptoms like psychosis, depression and paranoia are also risk factors for falls. The medications used for management of these symptoms such as SSRI, neuroleptics and benzodiazepines are also known to increase fall risk. [22-26]

The use of physical restraints is a common practice in acute settings for the elderly who are restless, agitated and are unable to follow instructions. The indications for physical restraint use include managing and controlling the behavioural symptoms in order to reduce falls and prevent dislodgement of medical devices. Restraints have not been shown to reduce falls among the elderly. On the contrary, the use of restraints have been linked to serious injurious falls and complications arising from immobility such as delirium, cognitive decline, urinary retention, constipation, etc. Physical restraints should be considered as the last resort where non-pharmacological management of behavioural symptoms fail or the elderly is of danger to him/her self and others surrounding them. [27]

Urinary Incontinence and fall risk: Urinary incontinence [UI] is common among the elderly patients with dementia, with urge incontinence being the commonest type. UI in dementia may be due to autonomic dysfunction, loss of central control or due to functional causes. UI is known to increase fall risk among the elderly with dementia due to frequent needs to rush to toilet, association with orthostatic hypotension, especially if there is autonomic dysfunction associated with Lewy Body dementia or Parkinson's disease dementia. Medication used in the management of $\mathrm{BPH}$ such as alpha-1 receptor blocker causes orthostatic hypotension and the older anticholinergic agents like Oxybutinin used in urge incontinence has the potential to cause delirium in the elderly. [28]

\section{Assessment of falls}

The American Geriatric Society and British Geriatric Society [2011] have a joined guideline which recommended that all elderly individuals should be routinely screened history of falls. If the elderly had previous history of falls, they need a detailed assessment of gait and balance. A clinician should perform a multifactorial assessment for the elderly who performed poorly on standardised gait and balance assessment.

Multifactorial assessment include a detailed history of fallsfrequency, circumstances of falls, injuries, symptoms prior to falls and screened for comorbidities which may have precipitated the falls. A detailed medication review is also indicated.

A thorough physical examination of the cardiovascular system for rhythm abnormalities, pulse rate, blood pressure and orthostatic hypotension are essential. Neurological examination, particularly lower limb muscle strength, tone, proprioception, reflexes are also recommended. Tests of cortical, extrapyramidal and cerebellar functions may indicate the cause of gait abnormalities. Lower limb arthropathy, feet deformities and footwear may also contribute to falls.

Functional assessment includes patients' abilities to carry out their ADLs with or without aids, and for the elderly with risk taking behaviours, it is useful to assess if they have insight into their limitations. Fear of falling may contribute to self-imposed restrictions in activities which predisposes to functional decline. Home environmental assessment is useful for selected individuals.

Treatment which have been shown to reduce falls include vitamin D supplement of $>800$ IU a day for the elderly with high fall risk or those with proven vitamin D deficiency. [29]

\section{General strategies to reduce falls for the elderly}

Most of the recommendations were based on results from trials done on community dwelling elderly and these trials excluded elderly with cognitive impairment.

For the general approach to form interventions for the elderly fallers, the risk factors for falls should be clearly identified. The interventions should be individualized, together with an exercise programme. Recommendations for effective fall risk reduction for a relatively fit, community dwelling elderly include, modifications of home environment, medication review with an aim to minimise and remove unnecessary medications, particularly psychotropics [antidepressants, sedatives and antipsychotics], management of orthostatic hypotension and proper advice on footwear and foot problems. Exercise programmes, which may be group or individualized home programmes, consist of muscle strengthening, gait and balance training. Tai Chi is helpful in balance training and fall reduction. A referral to physical therapist is recommended for prescription of exercises. Exercise programme should ideally be prescribed taking into account the individual's functional and health profiles. [29]

The elderly patients with Alzheimer's Disease are more likely to sustain hip fractures after a fall. The other risk factors for osteoporosis should be screened for and intervene as appropriate. It may be helpful to screen for osteoporosis and institute treatment to reduce fracture risk among the elderly with frequent falls. [30]

\section{Strategies to reduce falls among the elderly with dementia or cognitive impairment}

For the elderly with delirium in the acute hospital setting, routine assessment for fall risk and mental state is important to reduce hospital falls. This include putting up routine falls precaution, diagnosis of delirium and background cognitive issues. The elderly patients with cognitive impairment and delirium have difficulties retaining information and instructions and their care needs are higher. Once cognitive issues are identified, supportive care such as reorientation, ensuring regular sleep pattern [environmental factors and sleep hygiene], using appropriate sensory aids [hearing aids and glasses], adequate pain control, monitor elimination needs, regular ambulation and implementing a daily routine are all helpful to reduce in-patient delirium and falls. [23] 
There is currently insufficient evidence for interventions to reduce falls specifically among the elderly with dementia or cognitive impairment in the community or living in long term care. Shaw and colleagues were unable to show benefits of multifactorial interventions in fall prevention among the elderly with dementia presenting to the emergency department. [31]

For the older people with dementia who fall, they tend to be older, female and more likely to be living in an institution. They also tend to have more severe impairment of gait and balance, which is often not due to ageing alone, but rather central causes for the abnormal gait. [732] The elderly with dementia are also more likely to have autonomic dysfunction causing orthostatic hypotension contributing to falls. [33] The elderly with dementia often have behavioural symptoms and the pharmacological interventions for these symptoms too, increase fall risk, as mentioned above.

The elderly with dementia were able to comply with prescribed interventions like modifications of gait and balance, environmental factors, medications and exercise programmes. The effect of interventions on fall reduction was observed to be more effective among the elderly with higher MMSE scores compared to the elderly with lower scores. [31-34]

In summary, most of the recommendations for multifactorial interventions for fall reductions are effective for the fitter, cognitively intact elderly. The elderly with dementia failed to show significant improvements with the conventional recommendations. The lack of effectiveness could be because dementia carries with it a different set of risk factors which have not been nevertheless, the elderly fallers should be actively screened for risk factors for falls and interventions put in as appropriate.

\section{References}

1. The Global Voice on Dementia. Alzheimer's Disease Association International. https:// www.alz.co.uk/research/statistics

2. Tinetti ME1, Doucette J, Claus E, Marottoli R (1995) Risk factors for serious injury during falls by older persons in the community. J Am Geriatr Soc 43: 1214-1221. [Crossref]

3. Oleske DM1, Wilson RS, Bernard BA, Evans DA, Terman EW (1995) Epidemiology of injury in people with Alzheimer's disease. J Am Geriatr Soc 43: 741-746. [Crossref]

4. Tinetti ME1, Williams CS (1998) The effect of falls and fall injuries on functioning in community-dwelling older persons. J Gerontol A Biol Sci Med Sci 53: M112-119. [Crossref]

5. Oliver D1, Daly F, Martin FC, McMurdo ME (2004) Risk factors and risk assessment tools for falls in hospital in-patients: a systematic review. Age Ageing 33: 122-130. [Crossref]

6. Kurrle, S., Brodaty, H., \& Hogarth, R (2012) Falls.In Physical Comorbidities of Dementia [pp.3-14]. Cambridge: Cambridge University Press. doi:10.1017/ CBO9780511894534.004

7. Elble RJ1 (2007) Gait and dementia: moving beyond the notion of gait apraxia. $J$ Neural Transm (Vienna) 114: 1253-1258 [Crossref]

8. Atkinson HH, Rosano C, Simonsick EM (2007) Cognitive function, gait speed decline, and comorbidities: The health, aging and body composition study. J Gerontol A Biol Sci Med Sci 62: 844-850 [Crossref]

9. Mielke MM, Roberts RO, Savica R (2013) Assessing the temporal relationship between cognition and gait: slow gait predicts cognitive decline in the Mayo Clinic Study of Aging. J Gerontol A Biol Sci Med Sci 68: 929-937 [Crossref]

10. Julien Durmurgier (2017) Gait speed and decline in gait speed as predictors of incident dementia. J Gerontol A Biol Sci Med Sci 72: 655-661 [Crossref]

11. Baezner H (2008) Association of gait and balance disorders with age related white matter changes: the LADIS study. Neurology 70: 935-42. [Crossref]
12. Allan LM1, Ballard CG, Burn DJ, Kenny RA (2005) Prevalence and severity of gait disorders in Alzheimer's and non-Alzheimer's dementias. J Am Geriatr Soc 53: 16811687. [Crossref]

13. Waite L (2000) Motor function and disability in the dementias. International Journal of Geriatric Psychiatry [15]: 897 -903. [Crossref]

14. O. Beauchet (2009) Stops walking when talking: a predictor of falls in older adults? European Journal of Neurology 16: 786-795. [Crossref]

15. Kressig RW1, Herrmann FR, Grandjean R, Michel JP, Beauchet O (2008) Gait variability while dual-tasking: fall predictor in older inpatients? Aging Clin Exp Res 20: 123-130. [Crossref]

16. Johansson J1, Nordström A2, Nordström P3 (2016) Greater Fall Risk in Elderly Women Than in Men Is Associated With Increased Gait Variability During Multitasking. $J$ Am Med Dir Assoc 17: 535-540. [Crossref]

17. Panayotes Demakakos (2013) the bidirectional association betweeb depressive symptoms and gait speed: Evidence from the English Longitudinal Study of Ageing [ELSA]. PLoS One 87: e68632 [Crossref]

18. Sophia Bennett, AJ Thomas (2014) Depression and dementia: cause, consequence or coincidence. Maturitas 79: 184-190 [Crossref]

19. Lien Quach (2013) Depression, antidepressants and falls among community dwelling elderly people: the MOBILIZE study. The Journals of Gerontology 68: 1575-1581 [Crossref]

20. Vaughan L1, Corbin AL1, Goveas JS2 (2015) Depression and frailty in later life: a systematic review. Clin Interv Aging 10: 1947-1958. [Crossref]

21. Beijer HJ1, de Blaey CJ (2002) Hospitalisations caused by adverse drug reactions (ADR): a meta-analysis of observational studies. Pharm World Sci 24: 46-54 [Crossref]

22. American geriatrics Society (2015) Updated Beers Criteria for potentially inappropriate medications use in older adults. JAGS 63: 2227-2246. [Crossref]

23. Lakatos BE (2009) Falls in general hospital: association with delirium, advanced age and specific surgical procedures. Psychosomatics 50: 218-226 [Crossref]

24. Mahoney J (2000) Temporal association between hospitalisation and rate of falls after discharge. Arch Intern Med 160: 2788-2795. [Crossref]

25. Flaherty JH1, Morley JE (2013) Delirium in the nursing home. J Am Med Dir Assoc 14: 632-634 [Crossref]

26. Kallin K (2005) Factors associated with falls among older, cognitively impaired people in geriatric care settings. A population based study. Am J Geriatr Psychiatry 13: 501 509. [Crossref]

27. Lim SC (2016) Restraint use in the management of elderly with dementia in hospital. Internal Med Res Open J 1: 1-4

28. SC Lim (2017) Managing the elderly with urinary incontinence and dementia. Int Arch Urol Complic 3: 027.

29. (2011) AGS/BGS Clinical Practice Guideline for prevention of falls. JAGS January 59: 1 .

30. Iris Weller (2004) Hip fractures and Alzheimer's disease in elderly institutionalized Canadians. Annals of Epidemiology 14: 319-324. [Crossref]

31. Shaw FE (2003) Multifactorial intervention after a fall in older people with cognitive impairment and dementia presenting to the accident and emergency department: randomized control trial. Br Med J 326: $73-75$ [Crossref]

32. Waite LM1, Broe GA, Grayson DA, Creasey H (2000) Motor function and disability in the dementias. Int J Geriatr Psychiatry 15: 897-903. [Crossref]

33. Idiaquez J1, Roman GC (2011) Autonomic dysfunction in neurodegenerative dementias. J Neurol Sci 305: 22-27. [Crossref]

34. Jensen J1, Nyberg L, Gustafson Y, Lundin-Olsson L (2003) Fall and injury prevention in residential care--effects in residents with higher and lower levels of cognition. $J \mathrm{Am}$ Geriatr Soc 51: 627-635. [Crossref]

Copyright: (C2017 Si Ching LIM. This is an open-access article distributed under the terms of the Creative Commons Attribution License, which permits unrestricted use, distribution, and reproduction in any medium, provided the original author and source are credited. 